\title{
BMJ Open Community-based weight loss programme targeting overweight Chinese adults with pre-diabetes: study protocol of a randomised controlled trial
}

\author{
Mandy Ho (D) , ${ }^{1}$ Pui Hing Chau, ${ }^{1}$ Esther Yee Tak $\mathrm{Yu},{ }^{2}$ Michael Tin-cheung Ying, ${ }^{3}$ \\ Cindy Lo Kuen Lam²
}

To cite: $\mathrm{Ho}$ M, Chau PH, Yu EYT, et al. Communitybased weight loss programme targeting overweight Chinese adults with pre-diabetes: study protocol of a randomised controlled trial. BMJ Open 2020;10:e035196. doi:10.1136/ bmjopen-2019-035196

- Prepublication history for this paper is available online. To view these files, please visit the journal online (http://dx.doi. org/10.1136/bmjopen-2019035196).

Received 23 0ctober 2019 Revised 17 March 2020 Accepted 19 March 2020

Check for updates

(C) Author(s) (or their employer(s)) 2020. Re-use permitted under CC BY-NC. No commercial re-use. See rights and permissions. Published by BMJ.

${ }^{1}$ School of Nursing, University of Hong Kong Li Ka Shing Faculty of Medicine, Pokfulam, Hong Kong

${ }^{2}$ Department of Family Medicine and Primary Care, University of Hong Kong Li Ka Shing Faculty of Medicine, Pokfulam, Hong

Kong

${ }^{3}$ Department of Health

Technology and Informatics, Hong Kong Polytechnic University, Kowloon, Hong Kong

Correspondence to

Dr Mandy Ho;

mandyho1@hku.hk

\section{ABSTRACT}

Introduction Type 2 diabetes mellitus (T2DM) is one of the world's fastest growing health problems. Asians have a strong ethnic predisposition for T2DM, developing T2DM at a lower degree of obesity and at younger ages than other ethnic groups. T2DM has a gradual onset, with most individuals progressing through a pre-diabetic state, providing an opportunity to prevent T2DM and its complications. This study aims to evaluate the effectiveness of a community-based lifestyle intervention programme on weight loss and improvements in insulin sensitivity and cardiometabolic profiles in Chinese adults with pre-diabetes. Methods and analysis This study is a 12-month, assessor-blinded randomised controlled trial. Adults with pre-diabetes (aged 40-64 years, $\mathrm{n}=180$ ) with pre-diabetes are randomised into either an intervention group (receiving group-based lifestyle interventions) or a control group (receiving text messages containing health information). The intervention programme targets a weight loss of $5 \%$ during the first 6 months by restricting caloric intake and increasing physical activity. Participants in the intervention group will attend six group sessions and two individual face-to-face diet counselling sessions during the first 6 months, followed by monthly telephone support during the 6-month maintenance phase. Participants in the control group will receive monthly text messages containing general health information only. The primary outcome is weight loss (\%). Secondary outcomes include insulin sensitivity (assessed using fasting insulin level and homeostatic model assessment of insulin resistance), glycaemic control (assessed using glycated haemoglobin level), lipid profile, blood pressure, carotid artery thickness, dietary intake and level of physical activity. Intention-to-treat analysis will be conducted using a generalised linear mixed effects model with a logit link and linear mixed models.

Ethics and dissemination This study has been approved by the relevant research ethics committee. The results will be disseminated through peer-reviewed journals and scientific presentations.

Trial registration number NCT03609697.

\section{INTRODUCTION}

Type 2 diabetes mellitus (T2DM) is a major non-communicable disease and one of the world's fastest growing health problems. In
Strengths and limitations of this study

- This is the first randomised controlled trial to evaluate the effectiveness of a community-based lifestyle intervention programme on weight loss and improvements in insulin sensitivity and cardiometabolic profiles in Chinese adults with pre-diabetes.

- This weight loss intervention programme is specifically tailored to the Chinese culture and contemporary Hong Kong context.

- The findings of this study will provide an evidence base for the development of an effective type 2 diabetes mellitus prevention model in Asian countries.

- Owing to the nature of the intervention, it is not possible to blind the participants or interventionists.

2013, more than 380 million people were affected worldwide, and this number is expected to rise to 592 million by 2035 and many cases are undiagnosed. ${ }^{1}$ T2DM is associated with significant morbidity, including increased risk of cardiovascular diseases (CVD) and stroke, hypertension, retinopathy and blindness, renal failure, and leg amputation. These place an enormous burden on individuals, society and the healthcare system. $^{2}$ The Asia-Pacific region carries a particularly high disease burden, with more than $60 \%$ of the global diabetic population living in the Asian region. ${ }^{3}$ Asians have a strong ethnic predisposition for T2DM and develop T2DM at a lower degree of obesity and at younger ages than other population groups. ${ }^{4}$

T2DM is a non-reversible but preventable condition. The onset of T2DM is gradual, with most individuals progressing from normoglycaemia through a pre-diabetic state. People with pre-diabetes, defined as having impaired fasting glucose (IFG), impaired glucose tolerance (IGT) or impaired glycated haemoglobin $(\mathrm{HbA1c}),{ }^{2}$ are at increased risk 
of developing T2DM and its associated complications, such as CVD and retinopathy, which can develop even in the absence of progression to overt T2DM. ${ }^{5-7}$ Hence, it is essential that people with pre-diabetes are targeted for early intervention to prevent T2DM and related complications. A national survey conducted in China in 2010 revealed that $50.1 \%$ of people aged 18 years or older have pre-diabetes. ${ }^{8}$ Pre-diabetes is a prevalent and potentially reversible condition. It provides an important window of opportunity for healthcare providers to implement interventions that can delay or prevent T2DM and its complications. Of particular concern is that evidence suggests that, without targeted intervention, the progression from pre-diabetes to T2DM is faster in Asians than in Caucasians. ${ }^{910}$

Overweight and obesity are major risk factors for T2DM. International clinical trials have demonstrated that lifestyle interventions targeting at least $5 \%$ weight loss in individuals with pre-diabetes can be cost-effective in preventing T2DM. ${ }^{11}$ Lifestyle interventions (which include diet, physical activity and behavioural modification components) and self-management of pre-diabetes have been listed as effective means of decreasing the incidence of T2DM in the international guidelines on the management of pre-diabetes. ${ }^{679-13}$ The consensus statement from the American Diabetes Association on the management of pre-diabetes recommends lifestyle modification with a weight loss goal of 5\%-10\% and approximately $30 \mathrm{~min}$ of moderate-intensity physical activity daily. ${ }^{6}$ The Australian guidelines on the management of pre-diabetes recommend weight loss of at least 5\%-7\%, reduction of total fat intake to $<30 \%$ of total daily calories, reduction of saturated fat intake to $<10 \%$ of total daily calories, optimal dietary fibre intake and $150 \mathrm{~min}$ of physical activity per week.

Despite the recognised risk of individuals with prediabetes developing T2DM and the emerging evidence on the clinical and economic benefits of intervention at the pre-diabetes stage, data on the effectiveness of lifestyle intervention programmes targeting overweight Chinese adults with pre-diabetes in a local setting are limited. Approaches to translate diabetes prevention programmes into community settings have been investigated in Western countries, and demonstrated efficacy in achieving the weight loss goals and improving glycaemic control. ${ }^{14-16}$ Group-based interventions have been recommended as a cost-effective approach for community settings. To our knowledge, the effect of weight loss and/or lifestyle changes on subclinical atherosclerosis, assessed by changes in carotid artery intima-media thickness (cIMT), in the pre-diabetic population has not yet been explored. Successful interventions involving lifestyle changes tend to be context specific. Culture and social environment strongly influence people's attitudes and behaviours towards diet and physical activity. The results of overseas studies may not be applicable to Chinese adults in Hong Kong. The Da Qing IGT and Diabetes Study is the only large randomised controlled trial (RCT) to date which has targeted the Chinese population; however, this intervention programme was conducted from 1986 to 1992, more than 25 years ago. ${ }^{17}$ Hence, this study aims to translate evidence-based diabetes prevention strategies into a community setting and to evaluate the effect of a community-based lifestyle intervention programme on weight loss and improvements in insulin sensitivity and metabolic profiles in Chinese adults with pre-diabetes. This study will develop resources on diet and physical activity that are tailored to the Chinese culture and contemporary Hong Kong context. Determining which approaches to lifestyle changes are effective within the contemporary Hong Kong context will be valuable for developing appropriate and effective diabetes prevention interventions for Chinese and Asian populations.

\section{OBJECTIVES}

1. To evaluate the effect of a community-based lifestyle intervention programme on weight loss in Chinese overweight adults with pre-diabetes, compared with minimal intervention (receiving short message service (SMS) messages containing health information).

2. To evaluate the effect of a community-based lifestyle intervention programme on insulin sensitivity, glycaemic control, cardiometabolic profiles (blood pressure and lipid profile), health-related quality of life (QoL) and cIMT in Chinese overweight adults with pre-diabetes, compared with minimal intervention (receiving SMS messages containing health information).

\section{METHODS AND ANALYSIS}

The study is being conducted as a 12-month, assessorblinded RCT (performed according to the Consolidated Standards of Reporting Trials guidelines) of communitybased lifestyle intervention, including two study arms:

1. Intervention group (receiving group-based lifestyle interventions).

2. Control group (receiving SMS intervention).

Figure 1 illustrates the pathway through the trial. Ethical approval was obtained from the Institutional Review Board (IRB) of the Hong Kong West Cluster, the Hospital Authority in Hong Kong (UW18-203). Written informed consent has been obtained from all participants, and the RCT was registered with ClinicalTrials.gov (registered on 1 August 2018).

\section{Participants}

Previous studies indicated that a community-based T2DM prevention programme would achieve greater health and economic gains if it was directed at people under the age of $65 .{ }^{18}$ Hence, this study targets people aged $40-65$ years.

\section{Inclusion criteria}

Chinese adults aged between 40 and 65 years, who were overweight (body mass index (BMI) $\geq 23 \mathrm{~kg} / \mathrm{m}^{2}$ ) or obese (BMI $\geq 25 \mathrm{~kg} / \mathrm{m}^{2}$ ), with IGT (blood glucoselevel of $7.8-11.0 \mathrm{mmol} / \mathrm{L}$ after a 2-hour oral glucose tolerance test (OGTT)), IFG 


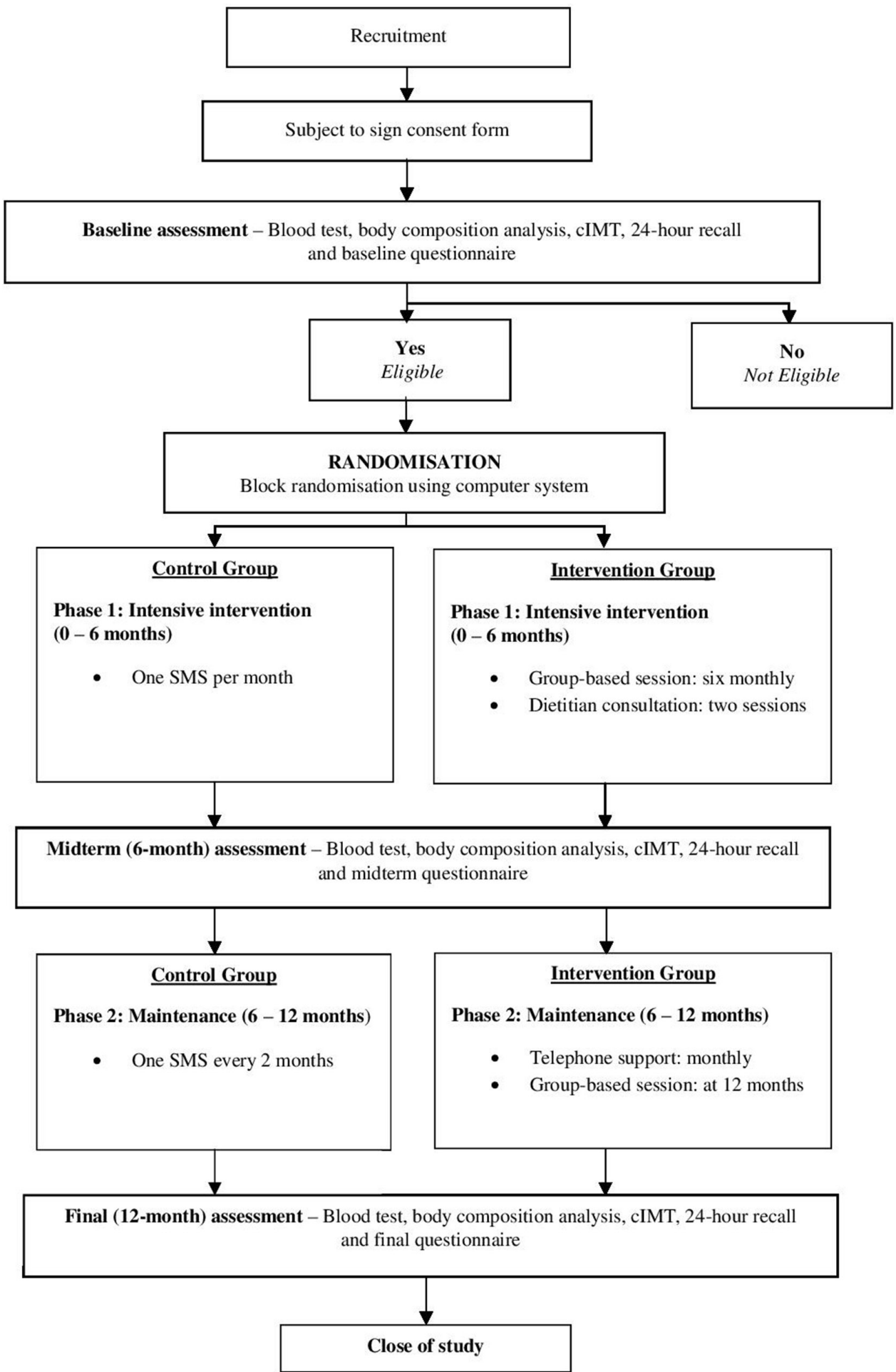

Figure 1 Flow chart of intervention and participant involvement throughout the trial. cIMT, carotid artery intima-media thickness; SMS, short message service.

(fasting blood glucose level of $5.6-6.9 \mathrm{mmol} / \mathrm{L}$ ) or impaired HbAlc (HbA1c level of 5.7\%-6.4\%) were eligible to participate. ${ }^{2}$ For intervention purposes, eligible participants were also required to have a mobile phone and be able to read Chinese and speak Cantonese.

\section{Exclusion criteria}

People with a current diagnosis or clinical history of T2DM, or with comorbid conditions that may limit participation in the study, such as a history of an acute cardiovascular event, uncontrolled hypertension, cancer or major psychiatric or cognitive problems, were excluded. People who were already participating in a weight loss programme, were receiving drug treatment for pre-diabetes (eg, metformin) or had a history of longterm use of medicines known to influence glucose metabolism (eg, corticosteroids) were also ineligible. 


\section{Recruitment}

Participants were recruited from the community by convenience sampling between August 2018 and March 2019. A study hotline was set up for interested individuals to obtain basic information about participation and the required commitment for participation in the study. Potential participants underwent OGTT and HbA1c tests to confirm their eligibility and pre-diabetic status (IFG, IGT or impaired HbA1c). The American Diabetes Association diagnostic criteria for pre-diabetes and T2DM are used throughout this study. ${ }^{2}$

\section{Sample size}

The estimated minimum required sample size was based on the primary outcome: the proportion of participants achieving $5 \%$ weight loss between the baseline assessment and 12 months. Previous lifestyle intervention trials have reported that around $50 \%$ of participants had lost at least $5 \%$ of their baseline body weight at 12 months. ${ }^{16} \mathrm{We}$ predicted that weight loss in the control group (receiving minimal intervention) would be half of that in the intervention group; that is, $25 \%$ of participants in the control group may lose at least $5 \%$ of their baseline body weight at 12 months. To detect this difference in the proportion of participants achieving $5 \%$ weight loss between the intervention and control groups, 58 participants were required in each group, to ensure $80 \%$ power at a $5 \%$ significance level. To accommodate a $35 \%$ dropout rate, ${ }^{14}$ we therefore needed to recruit 90 participants in each intervention group (180 in total). This was based on a conservative estimate: if fewer than $25 \%$ of participants in the control group achieve $5 \%$ weight loss, the required sample size will be less than 180 .

\section{Randomisation and blinding}

Eligible participants were randomised into either the SMS intervention group (control group) or the communitybased lifestyle intervention group (intervention group) by block randomisation with block sizes of 4, 6 and 8 , using a computer program. Intervention assignments were concealed in sequentially numbered, opaque, sealed envelopes prepared by a statistician who was not directly involved with the study. The intervention assignment was revealed after the consent form was signed by the study participant. Due to the nature of the study, we were not able to blind participants to their intervention assignments. However, blinding of outcome assessors is being applied. All blood tests are being collected and analysed by an accredited laboratory that is blinded to the intervention allocation. Anthropometric, dietary and cIMT measurements are being taken by trained researchers who are not involved in any part of the intervention and are blinded to the intervention allocations.

\section{Intervention group}

This study has adopted key aspects of the successful Diabetes Prevention Program, ${ }^{11}$ with adaptations made for the Chinese culture and contemporary Hong Kong context. The primary goal of the intervention is to achieve a weight reduction of at least $5 \%$ of baseline body weight during the first 6 months, through restriction of caloric intake (goals: 1200-2000 kcal/day, $<30 \%$ of total daily calories from fat and $<10 \%$ of total daily calories from saturated fat) and increased physical activity (goal: $\geq 150$ min of physical activity per week). This approach is consistent with the recommendations of the international guidelines on the management of pre-diabetes. ${ }^{619}$ Table 1 shows a summary of the intervention programme. The intervention will be based on the health action process approach, which aims to motivate health behaviour changes in participants and to develop their goal setting, self-monitoring, problem solving and decision-making skills. ${ }^{20}$ At baseline, all participants will be provided with a logbook to record their individualised goals and progress, including body weight, waist circumference, blood pressure, blood lipids and blood glucose levels, and to maintain physical activity and diet diaries throughout the study period. Tailored resources on diet and physical activity are being developed and provided throughout the intervention period to suit the Chinese culture and Hong Kong context. At baseline, each participant was assigned a nursing student to act as a health buddy. The health buddies attend all group-based intervention sessions with the participants during the active intervention phase, to establish rapport and provide support to the participants. Training and ongoing coaching is provided by the study investigators to empower the health buddies with knowledge and skills to support individuals with pre-diabetes to achieve their weight loss goals. The health buddies are responsible for supporting the participants during group sessions and providing telephone support to participants during the maintenance phase, under the guidance and supervision of the research team.

The programme is being conducted in small groups of 10-12 participants, facilitated by a trained healthcare professional. There are eight groups, located in various districts of Hong Kong. Participants can choose a location close to where they live or work. The programme consists of two phases: phase 1 (months $0-6$ ) is the intensive intervention phase, and phase 2 (months $7-12$ ) is the maintenance phase.

During phase 1, participants are expected to attend monthly group sessions (2 hours per session, six sessions in total). The group sessions involve education about prediabetes self-management, weight loss, nutrition, physical activity, goal setting, self-monitoring, problem solving, decision-making and engagement in long-term lifestyle modification (table 1). Participants are encouraged to increase physical activity and reduce their sedentary lifestyle. Each participant has been given a pedometer to facilitate goal setting and self-monitoring of physical activity. Participants are encouraged to walk 8000-10 000 steps/day.

Participants also receive two individual face-to-face sessions with a dietitian. The dietitian estimates the daily energy requirements for each participant, according to their age, gender and physical activity levels. A low-fat diet 
Table 1 Summary of the 12-month lifestyle intervention programme for the intervention group

\begin{tabular}{|c|c|c|c|}
\hline Phase & Setting & Contact schedule & Objectives \\
\hline $\begin{array}{l}\text { Phase } 1 \\
\text { (months 1-6) } \\
\text { Intensive intervention }\end{array}$ & $\begin{array}{l}\text { Group } \\
\text { (10-12 participants) } \\
\text { One to one with } \\
\text { dietitian }\end{array}$ & $\begin{array}{l}\text { Six sessions } \\
\text { (monthly, } 2 \text { hours/ } \\
\text { session) } \\
\text { Two sessions } \\
\text { (1 hour/session) }\end{array}$ & $\begin{array}{l}\text { Knowledge: pre-diabetes self-management; diabetes risk } \\
\text { and progression; relation of overweight to disease and } \\
\text { health; benefits and importance of weight loss and lifestyle } \\
\text { modification; principles and methods of healthy weight } \\
\text { loss; and diet and physical activity for managing pre- } \\
\text { diabetes and preventing diabetes. } \\
\text { Diet: restriction of caloric intake (goals: } 1200-2000 \mathrm{kcal} / \\
\text { day, <30\% of total daily calories from fat and }<10 \% \text { of } \\
\text { total daily calories from saturated fat). } \\
\text { Physical activity: reduce sedentary activities; gradual } \\
\text { progression to } 150 \text { min of moderate intensity or equivalent } \\
\text { physical activity per week. } \\
\text { Behavioural skills: goal setting, self-monitoring, stimulus } \\
\text { control, problem solving, decision-making, relapse } \\
\text { management and social support. }\end{array}$ \\
\hline $\begin{array}{l}\text { Phase } 2 \\
\text { (months 7-12) } \\
\text { Maintenance }\end{array}$ & $\begin{array}{l}\text { Telephone } \\
\text { Group }\end{array}$ & $\begin{array}{l}\text { Monthly } \\
\text { One session at } 12 \\
\text { months } \\
\text { ( } 2 \text { hours) }\end{array}$ & $\begin{array}{l}\text { To provide support and reinforcement for continued effort } \\
\text { in behavioural management and weight management. } \\
\text { To reinforce skills learnt in phase } 1 \text { and encourage } \\
\text { engagement in long-term lifestyle modification and weight } \\
\text { management; to introduce community resources. }\end{array}$ \\
\hline
\end{tabular}

plan (with $<30 \%$ of total daily calories from fat and $<10 \%$ of total daily calories from saturated fat) is prescribed. The diet plan provides suggested servings of carbohydrates, proteins, fruits, vegetables and dairy products. The first session $(45 \mathrm{~min}$ ) is to discuss dietary goals and receive culturally appropriate and individualised dietary advice for weight loss and managing pre-diabetes. The second session $(30 \mathrm{~min})$ is to review progress and reinforce long-term dietary modifications.

During phase 2 (maintenance phase), participants receive monthly telephone contact from a health buddy, and a reinforcement group session is scheduled for 12 months. The purpose of monthly telephone contact is to provide support and reinforcement for continued effort in behavioural management (table 1).

\section{Control group}

For ethical reasons and to enhance the retention of participants, the control group is provided with SMS intervention, which is considered minimal intervention. During phase 1 , a text message is sent to participants once a month. During phase 2, a text message is sent every 2 months. The content of the messages will be related to general information about T2DM, pre-diabetes and lifestyle modification. The participants (both the intervention and control groups) are encouraged to continue to engage with their normal healthcare provided by their healthcare providers.

\section{Outcome measures}

Primary outcome

The primary outcome of this study is weight loss, as percentage weight loss is listed as the primary goal of lifestyle programmes in the international guidelines on the management of pre-diabetes. ${ }^{6}{ }^{19}$ Participants' weight is measured using standard procedures at baseline, 6 months and 12 months. Percentage weight loss from baseline is calculated.

\section{Secondary outcomes}

\section{Adiposity}

Body fat and waist circumference are measured using standard procedures at baseline, 6 months and 12 months.

\section{Blood tests}

An OGTT is performed after an overnight fast at baseline, 6 months and 12 months. In addition to glucose testing, fasting blood samples (drawn at time 0 in the OGTT) are also analysed for insulin, HbAlc and full lipid profiles. Insulin sensitivity is assessed using fasting insulin levels and homeostatic model assessment of insulin resistance $($ HOMA-IR $=($ fasting plasma insulin concentration $(\mathrm{mIU} / \mathrm{L}) \times$ fasting glucose $(\mathrm{mmol} / \mathrm{L})) / 22.5)$. Glycaemic control is assessed using $\mathrm{HbAlc}$ and fasting glucose levels.

\section{Blood pressure}

Blood pressure is measured using an automated blood pressure monitor, according to standard procedures.

\section{Carotid artery intima-media thickness}

cIMT is a well-known marker of subclinical atherosclerosis and predictor of CVDs. It is measured using high-resolution B-mode ultrasonography, according to standard procedures. 


\section{Eating behaviour}

Dietary intake is assessed using 24-hour recalls at baseline, 6 months and 12 months. Dietary recalls are conducted by a trained research assistant who is blinded to intervention allocation. To assist with estimation of portion sizes, a food model book with picture guides of common foods and household measures, such as bowls, plates, glasses and spoons, is used. All records are checked and verified by a dietitian. Energy, protein, carbohydrate and fat intake are analysed using Food Processor Nutrition Analysis Software V.11.1 (ESHA Research, Salem, OR; 2015).

\section{Physical activity}

Physical activity levels are evaluated using the Chinese version of the International Physical Activity Questionnaire (IPAQ) short form. The IPAQ short form has been validated in Chinese adults in Hong Kong. ${ }^{21}$ The IPAQ short form contains seven items that require participants' recall of the frequency and duration of their physical activity levels 7 days prior to completing the form. It assesses physical activity at four different levels: vigorous intensity, moderate intensity, walking and sitting. ${ }^{22}$ The IPAQ is self-administered: participants fill out the IPAQ when they attend the ultrasound laboratory for cIMT measurement. If they have any questions on how to complete the IPAQ, they can seek help from the trained research assistants, who are blinded to intervention allocation.

\section{Health-related QoL}

Health-related QoL is assessed using the Chinese version of the 12-Item Short Form Health Survey (SF-12). The SF-12 consists of 12 items, which are categorised into two subscales: the physical component score (PCS) and the mental component score (MCS). The SF-12 PCS and MCS are calculated using standard algorithms, with a higher score indicating better QoL. ${ }^{23}$ The SF-12 is the most widely used tool for assessing health-related QoL and its validity and reliability has been confirmed in ethnic Chinese adults. $^{24}$

\section{Compliance}

Data regarding dietary intake and physical activity levels, together with attendance of the group-based programme and records of telephone support during the maintenance phase, are used to assess the compliance of participants in the intervention group.

\section{Incidence of diabetes}

The long-term goal of this study is to delay or prevent T2DM onset; hence, incidence of diabetes is also monitored.

\section{Data management and analysis}

IBM SPSS software for Windows V.23.0 (IBM) will be used to perform data analyses, with statistical significance set at $\mathrm{p}<0.05$. Primary analyses will be based on intention to treat in all randomised participants. Completer analysis will be performed for all randomised participants who received the intervention which they were originally allocated and who attended the baseline, 6-month and 12-month assessments. The proportions of participants achieving $5 \%$ weight loss will be compared between the intervention and control groups, using a generalised linear mixed effects model with logit link. Linear mixed models will be used to investigate changes in continuous variables (including weight, body fat, HOMA-IR, HbA1c, fasting insulin levels, cIMT, blood pressure and QoL scores) over time. The model will be built with intervention assignment as the between-subject factor, time as the within-subject factor and a group-by-time interaction. Potential covariates (age, sex, education level, baseline pre-diabetes status, weight, HbAlc, HOMA-IR, and so on) will be assessed and, if appropriate, incorporated into the model. In addition, the number of participants who have developed T2DM (defined as a fasting glucose level of $\geq 7.0 \mathrm{mmol} / \mathrm{L}$ or a 2-hour OGTT glucose level of $\geq 11.1 \mathrm{mmol} / \mathrm{L}$ ) at 12 months will be compared between groups using a logistic regression. ${ }^{2}$ Sensitivity analysis will be conducted to compare the findings of the intention-totreat analysis and completer analysis.

Data management will be handled by the School of Nursing of the University of Hong Kong, in accordance with the principles of Good Clinical Practice (GCP) and the Declaration of Helsinki.

\section{Patient and public involvement}

A pilot study was conducted before we launched this full-scale RCT. The participant representatives from the pilot study are involved in this study as volunteer helpers, assisting with the group education sessions during the intervention phase. Participants in this study will be invited to attend a sharing symposium and will receive a lay summary of the study findings.

\section{Trial status}

This study is currently in the intervention phase. The study began recruitment in June 2018, and interventions have been running since September 2018. Data collection will be completed by approximately August 2020.

\section{DISCUSSION}

T2DM is a major non-communicable disease and one of the world's fastest growing health problems. Pre-diabetes is a prevalent and potentially reversible condition, which provides an important opportunity for the prevention of T2DM and its complications. To the best of our knowledge, this study is the first RCT to evaluate the effectiveness of a community-based lifestyle intervention programme for promoting weight loss, improving insulin sensitivity and slowing the progression of subclinical atherosclerosis in Chinese overweight adults with pre-diabetes. The findings of this study will provide evidence base for the development of an effective T2DM prevention model in Asian countries, to alleviate the disease burden on individuals, the community and the healthcare system. 


\section{ETHICAL CONSIDERATIONS AND DISSEMINATION}

Ethical approval was obtained from the IRB of the Hong Kong West Cluster, the Hospital Authority in Hong Kong (UW18-203, approval granted 15 March 2018). The study is being conducted in accordance with the principles of GCP. The protocol and any amendments have been, and will be in the future, reviewed by the IRB of the Hong Kong West Cluster, the Hospital Authority in Hong Kong. Participants were made aware that participation in the study was voluntary and that they could withdraw from the study at any time without further explanation or any negative consequences. Participants were provided with a study information sheet and requested to sign a consent form before enrolment into the study. All data related to participants' personal information will be kept strictly confidential and anonymous with the use of code numbers. The results will be collectively collated, analysed and presented, and no identifying individual information will be reported. No serious adverse events (SAE) have been observed. If any SAEs occur, they will be reported to the IRB.

The findings of this study will be published in a peerreviewed journal and presented at relevant conferences, seminars and research meetings.

Contributors MH, PPHC and CLKL designed the study and acquired funding. MH, PPHC, EYTY and MTY oversee study implementation. MH prepared the first draft of the manuscript. All authors read, critically revised and approved the final version of the manuscript.

Funding This work was supported by the Health Care and Promotion Scheme under the Health and Medical Research Fund, Food and Health Bureau, Hong Kong (grant number 01170498).

Competing interests None declared.

Patient and public involvement Patients and/or the public were not involved in the design, or conduct, or reporting, or dissemination plans of this research.

Patient consent for publication Not required.

Provenance and peer review Not commissioned; externally peer reviewed.

Open access This is an open access article distributed in accordance with the Creative Commons Attribution Non Commercial (CC BY-NC 4.0) license, which permits others to distribute, remix, adapt, build upon this work non-commercially, and license their derivative works on different terms, provided the original work is properly cited, appropriate credit is given, any changes made indicated, and the use is non-commercial. See: http://creativecommons.org/licenses/by-nc/4.0/.

ORCID iD

Mandy Ho http://orcid.org/0000-0002-4460-7969

\section{REFERENCES}

1 Guariguata L, Whiting DR, Hambleton I, et al. Global estimates of diabetes prevalence for 2013 and projections for 2035. Diabetes Res Clin Pract 2014;103:137-49.

2 American Diabetes Association. Classification and diagnosis of diabetes. Diabetes Care 2015;38:S8-16.
3 Mirasol R, Thai AC, Ahmad A, et al. A consensus of key opinion leaders on the management of pre-diabetes in the Asia-Pacific region. Journal of the ASEAN Federation of Endocrine Societies 2017;32:6-12.

4 Yoon K-H, Lee J-H, Kim J-W, et al. Epidemic obesity and type 2 diabetes in Asia. Lancet 2006;368:1681-8.

5 Levitan EB, Song Y, Ford ES, et al. Is nondiabetic hyperglycemia a risk factor for cardiovascular disease? A meta-analysis of prospective studies. Arch Intern Med 2004;164:2147-55.

6 Nathan DM, Davidson MB, DeFronzo RA, et al. Impaired fasting glucose and impaired glucose tolerance: implications for care. Diabetes Care 2007;30:753-9.

7 Singleton JR, Smith AG, Russell JW, et al. Microvascular complications of impaired glucose tolerance. Diabetes 2003;52:2867-73.

$8 \mathrm{Xu} \mathrm{Y,} \mathrm{Wang} \mathrm{L,} \mathrm{He} \mathrm{J,} \mathrm{et} \mathrm{al.} \mathrm{Prevalence} \mathrm{and} \mathrm{control} \mathrm{of} \mathrm{diabetes} \mathrm{in}$ Chinese adults. JAMA 2013;310:948-59.

9 Pan XR, Li GW, Hu YH, et al. Effects of diet and exercise in preventing NIDDM in people with impaired glucose tolerance. The dA Qing IGT and diabetes study. Diabetes Care 1997;20:537-44.

10 Ramachandran A, Snehalatha C, Mary S, et al. The Indian diabetes prevention programme shows that lifestyle modification and metformin prevent type 2 diabetes in Asian Indian subjects with impaired glucose tolerance (IDPP-1). Diabetologia 2006;49:289-97.

11 Knowler WC, Barrett-Connor E, Fowler SE, et al. Reduction in the incidence of type 2 diabetes with lifestyle intervention or metformin. N Engl J Med 2002;346:393-403.

12 Gong Q, Gregg EW, Wang J, et al. Long-Term effects of a randomised trial of a 6-year lifestyle intervention in impaired glucose tolerance on diabetes-related microvascular complications: the China dA Qing diabetes prevention outcome study. Diabetologia 2011;54:300-7.

13 Lindstrom J, Peltonen M, Eriksson JG, et al. Improved lifestyle and decreased diabetes risk over 13 years: long-term follow-up of the randomised Finnish 2Program for Indians diabetes prevention demonstration project. Diabetes Care 2013.

14 Payne WR, Walsh KJ, Harvey JT, et al. Effect of a low-resourceintensive lifestyle modification program incorporating gymnasiumbased and home-based resistance training on type 2 diabetes risk in Australian adults. Diabetes Care 2008;31:2244-50.

15 Whittemore R, Melkus G, Wagner J, et al. Translating the diabetes prevention program to primary care: a pilot study. Nurs Res 2009;58:2-12.

16 Katula JA, Vitolins MZ, Rosenberger EL, et al. One-Year results of a community-based translation of the diabetes prevention program: Healthy-Living partnerships to prevent diabetes (help PD) project. Diabetes Care 2011;34:1451-7.

17 Pan XR, Li GW, Hu YH, et al. Effects of diet and exercise in preventing NIDDM in people with impaired glucose tolerance. The dA Qing IGT and diabetes study. Diabetes Care 1998;20:537-44.

18 Zhuo X, Zhang P, Gregg EW, et al. A nationwide community-based lifestyle program could delay or prevent type 2 diabetes cases and save $\$ 5.7$ billion in 25 years. Health Aff 2012;31:50-60.

19 Twigg SM, Kamp MC, Davis TM, et al. Prediabetes: a position statement from the Australian diabetes Society and Australian diabetes educators association. Med J Aust 2007;186:461-5.

20 Schwarzer R, Lippke S, Luszczynska A. Mechanisms of health behavior change in persons with chronic illness or disability: the health action process approach (HAPA). Rehabil Psychol 2011;56:161-70.

21 Macfarlane DJ, Lee CCY, Ho EYK, et al. Reliability and validity of the Chinese version of IPAQ (short, last 7 days). J Sci Med Sport 2007;10:45-51.

22 Lee PH, Macfarlane DJ, Lam TH, et al. Validity of the International physical activity questionnaire short form (IPAQ-SF): a systematic review. Int J Behav Nutr Phys Act 2011;8:115.

23 Ware J, Kosinski M, Keller SD. A 12-Item short-form health survey: construction of scales and preliminary tests of reliability and validity. Med Care 1996;34:220-33.

24 Lam CLK, Tse EYY, Gandek B. Is the standard SF-12 health survey valid and equivalent for a Chinese population? Qual Life Res 2005;14:539-47. 